\title{
因HAD
}

DOI: http://doi.org/10.22585/hospdomic.v3i2.60

\section{Consecuencias de la nutrición parenteral domiciliaria en adultos con síndrome de intestino corto: revisión exploratoria}

\section{Outcomes of home parenteral nutrition in adults with short bowel syndrome: scoping review}

David Campos Cañuelo', Javier Sanz-Valero ${ }^{2,3}$, Carmina Wanden-Berghe ${ }^{3,4}$.

1. Universidad Miguel Hernández. Facultad de Farmacia, Campus de Sant Joan d'Alacant. Alicante. España.

2. Universidad Miguel Hernández. Departamento de Salud Pública e Historia de la Ciencia, Campus de Sant Joan d'Alacant. Alicante. España.

3. Instituto de Investigación Sanitaria y Biomédica de Alicante (ISABIAL-FISABIO). Alicante. España.

4. Hospital General Universitario de Alicante. Unidad de Hospitalización a Domicilio. Alicante. España.

Correspondencia/Correspondence

Carmina Wanden-Berghe

carminaw@telefónica.net

Recibido/Received

10.11.2018

Aceptado/Accepted

16.02.2019
Conflicto de Intereses/Competing interest La autora y los autores de este trabajo declaran la inexistencia de conflicto de interés en el presente estudio.

CÓMO CITAR ESTE TRABAJO | HOW TO CITE THIS PAPER

Campos Cañuelo D, Sanz-Valero J, Wanden-Berghe C. Consecuencias de la nutrición parenteral domiciliaria en adultos con síndrome de intestino corto: revisión exploratoria. Hosp Domic. 20 19;3(2): 149-62. 


\section{RESUMEN}

Objetivo: Revisar la literatura científica relacionada con las consecuencias de la nutrición parenteral provista por los servicios de atención de la salud en el domicilio (NPD) en adultos con síndrome de intestino corto (SIC).

Método: Revisión exploratoria y sistemática de los artículos recuperados de las bases de datos bibliográficas MEDLINE (PubMed), The Cochrane Library, Embase, Scopus, Web of Science y LILACS, hasta septiembre de 2018. La ecuación de búsqueda se formuló mediante los descriptores "Parenteral Nutrition", "Short Bowel Syndrome" y "Home Care Services", utilizando también los Entry Terms relacionados y los filtros: «Humans», «Adult: 19+ years» y «Comparative Study» o "Clinical Trial». La calidad de los artículos se evaluó mediante el cuestionario CONSORT.

Resultados: De las 192 referencias recuperadas, tras aplicar los criterios de inclusión y exclusión, se seleccionaron 8 artículos: 6 ensayos clínicos y 2 estudios comparativos. De ellos 2 , artículos relacionaron la NPD en pacientes con SIC con su calidad de vida; 2 reportaron consecuencias derivadas de la asociación de un fármaco con la NPD (1 con teduglutida y 1 con hormona del crecimiento); 1 asoció el uso de teduglutida con la calidad de vida y 3 observaron algún tipo de complicación derivada del uso de la NPD.

Conclusiones: Se probó mejor calidad de vida en los pacientes que usaban bomba portátil. El uso de teduglutida resultó beneficioso para el equilibrio de líquidos y la absorción de monosacáridos, pero produjo un retraso en el tránsito intestinal sin efecto sobre el vaciado gástrico o la permeabilidad de la mucosa. La asociación de la hormona del crecimiento con la NPD mejoró la absorción intestinal. En los enfermos con SIC y NPD a largo plazo, una combinación de factores del huésped y factores ambientales aumentaban el riesgo de sepsis recurrente y además tenían una baja densidad mineral ósea que continuaba en la NPD prolongada. Serían necesarias futuras investigaciones, con una adecuada población, que aclaren la relación directa de los efectos de la NPD en adultos con SIC.
Palabras clave: Nutrición Parenteral; Apoyo Nutricional; Síndrome de Intestino Corto; Síndromes de Malabsorción; Complicaciones Posoperatorias; Servicios de Atención de Salud a Domicilio.

\section{ABSTRACT}

Objective: To review scientific literature related to the consequences of parenteral nutrition provided by health care services in the home (HPN) in adults with short bowel syndrome (SBS).

Method: Exploratory and systematic review of items recovered from the bibliographic databases MEDLINE (PubMed), The Cochrane Library, Embase, Scopus, Web of Science and LILACS, until September 2018. The search equation was formulated using the descriptors "Parenteral Nutrition", "Short BowelSyndrome" and "Home CareServices", also using the related EntryTerms and filters: «Humans», «Adult: 19 + years» and "ComparativeStudy» or «Clinical Trial». The quality of the articles was assessed using the CONSORT questionnaire.

Results: Of 192 recovered references, after applying the inclusion and exclusion criteria, we selected 8 articles: 6 clinical trials and $2 \mathrm{com}$ parative studies. Of these, 2 articles related the HPN in patients with SBS with their quality of life; 2 reported consequences derived from the association of a drug with the HPN (1 with teduglutida and 1 with growth hormone); 1 associated the use of teduglutida with the quality of life and 3 observed some kind of complication derived from the use of the HPN.

Conclusions: Better quality of life in patients who used portable pump was proved. The use of teduglutida was beneficial for fluid balance and the absorption of monosaccharides, but produced a retardation in the intestinal transit without effect on gastric emptying or mucosal permeability. The Association of the growth hormone with the HPN improved intestinal absorption In patients with SBS and HPN to long term, a combination of environmental factors and host factors increased the risk of recurrent sepsis and in addition they had a low bone mineral density that remained in extended HPN. Future research would be needed, with a proper population, which clarifies the direct relationship of the effects of the HPN in adults with SBS. 
Keywords: Parenteral Nutrition; Nutritional Support; Short Bowel Syndrome; Malabsorption Syndromes; Postoperative Complications; Home Care Services.

\section{INTRODUCCIÓN}

La insuficiencia intestinal es una complicación largamente reconocida asociada con el síndrome del intestino corto que produce una malabsorción después de una resección significativa del intestino, por muchas razones, o dismotilidad funcional (1).

La causa determinante del síndrome de intestino corto (SIC) es la resección masiva de intestino delgado, con o sin pérdida del colon, independientemente de la etiología que haya motivado dicha resección. La resección intestinal amplia produce suficientes alteraciones como para requerir soporte nutricional especializado. Las medidas básicas de tratamiento, especialmente en la fase aguda tras la resección intestinal o en presencia de complicaciones graves sobre pacientes con intestino corto, incluyen la repleción de fluidos y electrolitos y la instauración de soporte nutricional con el fin de prevenir la desnutrición. La nutrición enteral es el principal factor estimulador de la adaptación del intestino remanente. No obstante, su aplicación presenta dificultades en las fases agudas, por lo que los pacientes deben ser tratados frecuentemente con nutrición parenteral (2).

La presencia de desnutrición puede ser también de importancia en los pacientes con enfermedad inflamatoria intestinal. El soporte nutricional está indicado en estos casos como tratamiento primario de la enfermedad, como tratamiento de la desnutrición o como tratamiento perioperatorio en los pacientes que requieren cirugía (2).

Afortunadamente, el soporte nutricional y sobretodo la nutrición parenteral domiciliaria (NPD), ha permitido la supervivencia en la comunidad de aquellos pacientes con enfermedades graves resultantes en un fallo intestinal que hacía imposible su nutrición por otros métodos. La NPD está indicada si existe un fallo intestinal documentado (entendido como la reducción de la función intestinal al mínimo hasta el punto de que se requiere suplementación para mantener la salud y/o el crecimiento) con imposibilidad para la nutrición exclusiva por vía oral/enteral, posibilidad de manejo del enfermo en el domicilio y que no exista una expectativa corta de supervivencia, teniendo en cuenta la calidad de vida del paciente, el entorno familiar y la capacidad del enfermo y/o de sus cuidadores de entrenamiento para la terapia de NPD (3).

Es decir, la NPD está indicada cuando el tracto gastrointestinal es incapaz de mantener una nutrición e hidratación normales y el paciente está clínicamente estable y listo para ser dado de alta del hospital (4).

En esta línea, Winkler et al. (5), resaltaban la importancia de la NPD ya que proporciona nutrición e hidratación a los pacientes con síndrome de intestino corto e insuficiencia intestinal y, por lo tanto, es una terapia de mantenimiento de la vida para estos pacientes. Aunque, también señalan que existen múltiples factores que contribuyen a una menor calidad de vida en los pacientes dependientes de NPD, incluidos los temores de eventos adversos asociados, como las complicaciones relacionadas con el catéter, la enfermedad hepática asociada con la nutrición parenteral y la enfermedad ósea metabólica. Además, los pacientes dependientes de NPD informan que tienen problemas de sueño y fatiga diurna debido a ruidos de la bomba, alarmas del equipo y nicturia.

Ahora bien, los riesgos de la nutrición parenteral a largo plazo pueden ser limitados y la calidad de la prestación de servicios puede aumentar si la coordinación está en manos de un equipo sanitario especializado (6).

Queda patente, que en el SIC el adecuado apoyo nutricional y el correcto seguimiento son de suma importancia, y la morbilidad y mortalidad a largo plazo dependerán del reconocimiento y el 
manejo cuidadoso de las complicaciones asociadas (7). Y, a pesar de que el SIC es una enfermedad con una alta mortalidad y morbilidad desde que se ha establecido la NPD, hay un número creciente de pacientes que sobreviven a la pérdida aguda de la función intestinal (8).

Por todo lo anteriormente expuesto, este trabajo tuvo como objetivo revisar, con fines exploratorios, la literatura científica relacionada con las consecuencias de la nutrición parenteral provista por los servicios de atención de la salud en el domicilio en adultos con síndrome de intestino corto.

\section{MÉTODO}

\section{Diseño}

Estudio descriptivo transversal y análisis crítico de los trabajos recuperados mediante revisión sistemática.

\section{Fuente de obtención de los datos}

Los datos se obtuvieron de la consulta directa y acceso, vía Internet, a las siguientes bases de datos bibliográficas del ámbito de las ciencias de la salud: MEDLINE (vía PubMed), The Cochrane Library, Embase, Scopus, Web of Science y LILACS

\section{Tratamiento de la información}

Para definir los términos de la búsqueda se consultó el Thesaurus desarrollado por la U.S. National Library of Medicine, conformando la sintaxis de búsqueda final mediante la intersección booleana de tres ecuaciones: (ecuación 1) AND (ecuación 2) AND (ecuación 3).

- Ecuación 1: Nutrición Parenteral - "Parenteral Nutrition"

"Parenteral Nutrition"[Mesh] OR "Parenteral Nutrition"[Title/Abstract] OR "Parenteral Feeding"[Title/Abstract] OR "Intravenous Feeding"[Title/Abstract] OR "Home Parenteral Nutrition" OR "Home Parenteral Feeding"[Title/Abstract] OR "Home Total Parenteral Nutrition"[Title/Abstract] OR "Parenteral Hyperalimentation"[Title/Abstract] OR "Total Parenteral Nutrition"[Title/Abstract] OR "Intravenous Hyperalimentation"[Title/Abstract]

- Ecuación 2: Síndrome del Intestino Corto - "Short Bowel Syndrome" "Short Bowel Syndrome"[Mesh] OR "Short Bowel Syndrome"[Title/Abstract]

- Ecuación 3: Servicios de Atención de Salud a Domicilio - "Home Care Services" "Home Care Services"[Mesh] OR "Home Care Service"[Title/Abstract] OR "Home Care Services"[Title/Abstract] OR "Home Care"[Title/Abstract] OR "Domiciliary Care"[Title/ Abstract] OR "Home Care Services, Hospital-Based"[Mesh] OR "Home Care Services, Hospital-Based" [Title/Abstract] OR "Hospital-Based Home Care"[Title/Abstract] OR "Hospital-Based Home Cares"[Title/Abstract] OR "Hospital Based Home Care"[Title/ Abstract] OR "Hospital Based Home Cares"[Title/Abstract] OR "Hospital Home Care Service"[Title/Abstract] OR "Hospital Home Care Services"[Title/Abstract] OR "Hospital-Based Home Care Services"[Title/Abstract] OR "Hospital Based Home Care Service"[Title/Abstract] OR "Hospital Based Home Care Services"[Title/Abstract] OR "Home Hospitalization"[Title/Abstract] OR "Hospital at Home"[Title/Abstract] OR "Hospitalat-Home"[Title/Abstract] OR "Hospital Home Care"[Title/Abstract] OR "Hospital Home 
Cares"[Title/Abstract] OR "Hospital at Home Care"[Title/Abstract] OR "Hospital at Home

Cares"[Title/Abstract] OR "Hospital in the Home"[Title/Abstract]

La ecuación de búsqueda final se desarrolló para su empleo en la base de datos MEDLINE, vía PubMed, utilizando los filtros: «Humans», «Adult: 19+ years» y "Comparative Study» o "Clinical Trial».

Esta estrategia se adaptó a las características de cada una del resto de bases de datos consultadas. La búsqueda se realizó desde la primera fecha disponible, de acuerdo a las características de cada base de datos, septiembre de 2018 y se completó con el examen del listado bibliográfico de los artículos que fueron seleccionados.

\section{Selección final de los artículos}

Se escogieron para su estudio los artículos que cumplieron los siguientes criterios: adecuarse a los objetivos de la búsqueda, estar publicados en revistas revisadas por pares y escritos en inglés, español, portugués, francés o alemán. La selección de los artículos pertinentes se realizó de forma independiente por dos autores: DCC y JSV. Para dar por válida la inclusión de los estudios se estableció que la valoración de la concordancia entre estos autores (índice Kappa) debía ser superior al $60 \%$ (fuerza de la concordancia buena). Siempre que se cumpliera esta condición, las posibles discordancias se solucionaron mediante la consulta a la autora CWB y posterior consenso entre todos los autores (9).

Para valorar la calidad de los documentos seleccionados se utilizaron las directrices para la comunicación de los ensayos clínicos CONSORT (Consolidated Standards of Reporting Trials) (10), que contiene un listado de 25 aspectos esenciales que deben describirse en la publicación de estos estudios. Para cada artículo seleccionado se asignó un punto por cada ítem presente (en caso de no ser aplicable no puntuaba). Cuando un ítem estaba compuesto por varios puntos, estos se evaluaron de forma independiente, dándole el mismo valor a cada uno de ellos y posteriormente se realizó un promedio (siendo éste el resultado final de ese ítem), de tal forma que en ningún caso se pudiera superar la puntuación de un punto por ítem.

\section{Extracción de los datos}

El control de la corrección de los datos se realizó mediante dobles tablas que permitieron la detección de las desviaciones y su subsanación mediante nueva consulta de los originales.

Para determinar la actualidad de los artículos se calculó el semiperíodo de Burton-Kebler (la mediana de la edad) y el Índice de Price (porcentaje de artículo con edad inferior a los 5 años).

Los estudios se agruparon según las variables a estudio, con el fin de sistematizar y facilitar la comprensión de los resultados, considerando los siguientes datos: primer autor de la referencia bibliográfica y año de publicación, tipo de estudio, población estudiada, país, tipo de nutrición, frecuencia de administración de la nutrición parenteral, existencia de fluidoterapia asociada a la nutrición, periodo en el que se realizó el estudio, resultado obtenido de la intervención.

\section{RESULTADOS}

Con los criterios de búsqueda descritos se recuperaron 192 referencias: 18 (9,38\%) en MEDLINE, 10 (5,21\%) en la Cochrane Library, $22(11,46 \%)$ en Embase, $127(66,15 \%)$ en Scopus y 15 $(7,81 \%)$ en la Web of Science. En la base bibliográfica LILACS no se recuperó ningún documento. 
Tras depurar los duplicados, aplicar los criterios de inclusión y exclusión, y consultar los listados bibliográficos de los artículos seleccionados (figura 1), fue posible seleccionar 8 documentos (11-18) para su revisión y análisis crítico (tabla 1).

\section{Figura 1 - Identificación y selección de estudios}
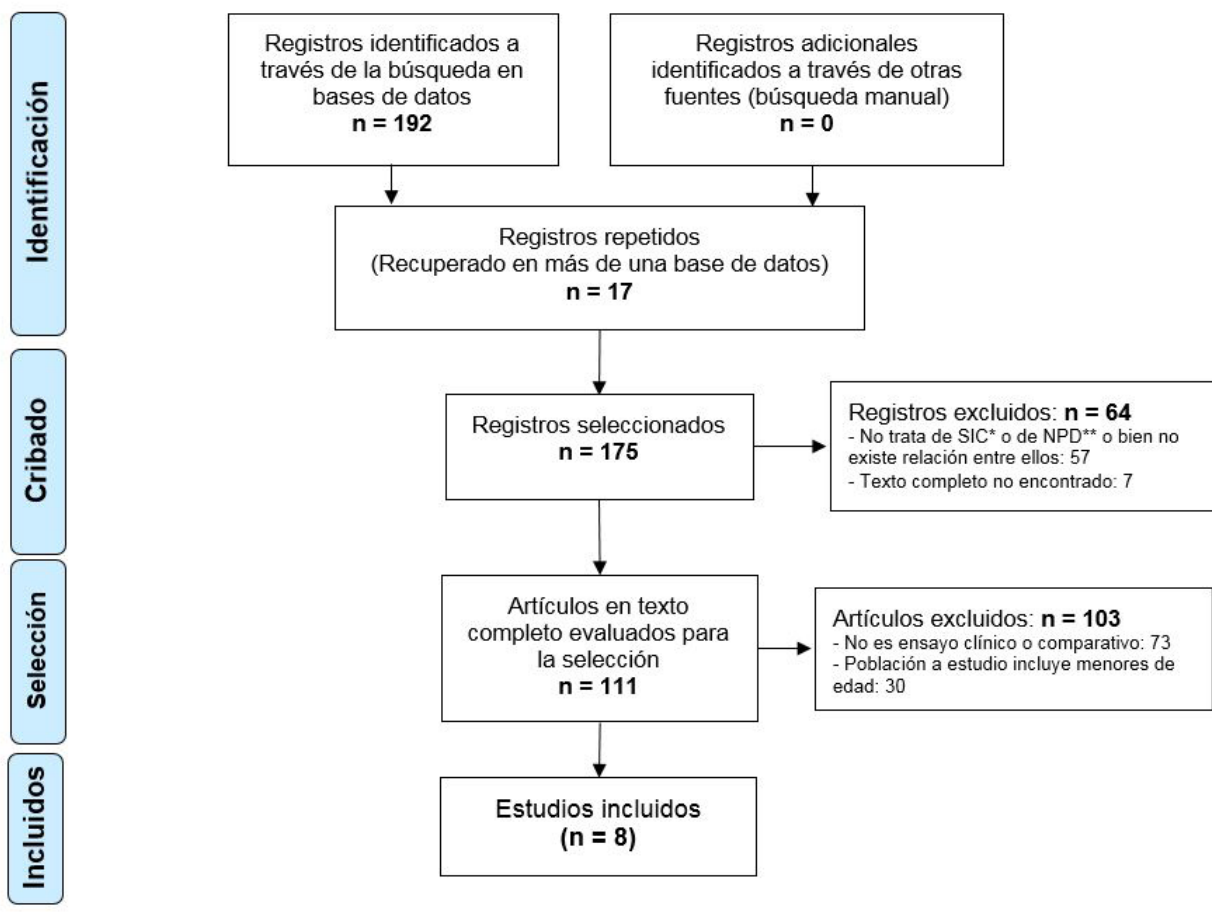

*SIC = Síndrome de Intestino Corto; ${ }^{\star \star}$ NPD = Nutrición Parenteral Domiciliaria

Tabla 1. Características de los estudios incluidos en la revisión sobre consecuencias de la nutrición parenteral domiciliaria en adultos con síndrome de intestino corto

\begin{tabular}{|c|c|c|c|c|c|c|c|}
\hline Autor, año & Diseño & Población & País & $\begin{array}{l}\text { Tipo de } \\
\text { nutrición } \\
\text { y Forma } \\
\text { administración }\end{array}$ & $\begin{array}{l}\text { Frecuencia de } \\
\text { administración } \\
\text { NPD y } \\
\text { Fármaco } \\
\text { asociado }\end{array}$ & Periodo & Resultado observado \\
\hline $\begin{array}{l}\text { Iturrino } \\
\text { et al. } \\
2016(11)\end{array}$ & $\begin{array}{l}\text { Ensayo } \\
\text { clínico }\end{array}$ & $\begin{array}{l}\text { Pt: } \\
N=8 ; 4 \mathrm{H} / 4 \mathrm{M} \\
\text { Gsic: } \\
N=8 ; 4 \mathrm{H} / 4 \mathrm{M}\end{array}$ & EEUU & $\begin{array}{l}\text { NPD } \\
\text { subcutánea }\end{array}$ & $\begin{array}{l}\text { Diaria } \\
(0,05 \mathrm{mg} / \mathrm{kg}) \\
\text { teduglutida }\end{array}$ & $\begin{array}{l}2 \text { años y } \\
2 \text { meses }\end{array}$ & $\begin{array}{l}\text { La administración de } \\
\text { teduglutida durante } 7 \\
\text { días resultó beneficioso } \\
\text { para el equilibrio de } \\
\text { líquidos y la absorción de } \\
\text { monosacáridos. } \\
\text { Produjo retraso en el } \\
\text { tránsito intestinal sin efecto } \\
\text { sobre el vaciado gástrico } \\
\text { y la permeabilidad de la } \\
\text { mucosa. }\end{array}$ \\
\hline
\end{tabular}




\begin{tabular}{|c|c|c|c|c|c|c|c|}
\hline $\begin{array}{l}\text { Saqui et al. } \\
2014 \text { (12) }\end{array}$ & $\begin{array}{l}\text { Ensayo } \\
\text { clínico }\end{array}$ & $\begin{array}{l}\text { Pt: } \\
\mathrm{N}=20 \\
5 \mathrm{H} / 15 \mathrm{M} \\
\text { Edad: } \\
52,8 \pm 3,3 \text { a. } \\
\text { Gsic:10 } \\
\text { Gc: } 10\end{array}$ & Canadá & $\begin{array}{l}\text { NPD } \\
\text { No consta }\end{array}$ & $\begin{array}{l}4,3 \pm 0.4 \text { veces } \\
\text { a la semana } \\
11,2 \pm 0,3 \\
\text { horas/dia } \\
\text { No consta }\end{array}$ & 2 meses & $\begin{array}{l}\text { Mayor facilidad de uso } \\
\text { y de calidad de vida } \\
\text { (SF-36) en los pacientes } \\
\text { que usaron bomba portátil } \\
\text { en comparación con una } \\
\text { bomba montada en poste. }\end{array}$ \\
\hline $\begin{array}{l}\text { Jeppesen } \\
\text { et al. } \\
2013 \text { (13) }\end{array}$ & $\begin{array}{l}\text { Ensayo } \\
\text { clínico }\end{array}$ & $\begin{array}{l}\text { Pt: } \\
\mathrm{N}=86 \\
\text { Grupos: } \\
\text { No consta }\end{array}$ & Dinamarca & $\begin{array}{l}\text { NPD } \\
\text { No consta }\end{array}$ & $\begin{array}{l}\text { No consta } \\
0,05 \mathrm{mg} / \mathrm{kg} / \mathrm{dia} \\
\text { teduglutida }\end{array}$ & 6 meses & $\begin{array}{l}\text { No se observó significación } \\
\text { entre los grupos } \\
\text { (medicamento vs. placebo) } \\
\text { en relación a la calidad de } \\
\text { vida (SBS-QoL) }\end{array}$ \\
\hline $\begin{array}{l}\text { Carlsson } \\
\text { et al. } \\
2004 \text { (14) }\end{array}$ & Comparativo & $\begin{array}{l}\text { Pt: } \\
N=19 ; \\
11 \mathrm{M} / 8 \mathrm{H} \\
\text { Edad }=54 \text { años } \\
\text { Gsic (NPD): } \\
\mathrm{N}=8 ; 4 \mathrm{H} / 4 \mathrm{M} \\
\text { Edad: } \\
50,3 \pm 6,6 \text { a. } \\
\text { Gc: } 11\end{array}$ & Suecia & $\begin{array}{l}\text { NPD } \\
\text { No consta }\end{array}$ & No consta & $\begin{array}{l}\text { Mediana } \\
\text { de } 74 \\
\text { meses; } \\
\text { rango } \\
61 \text { a } 201 \\
\text { meses }\end{array}$ & $\begin{array}{l}\text { La mayoría de los } \\
\text { pacientes mantuvieron una } \\
\text { condición clínica estable } \\
\text { dentro de los límites } \\
\text { normales de peso corporal } \\
\text { e IMC. } \\
\text { No se alteró ni la masa } \\
\text { libre de grasa ni el agua } \\
\text { corporal total en los } \\
\text { pacientes con NPD }\end{array}$ \\
\hline $\begin{array}{l}\text { Seguy et al. } \\
2003(15)\end{array}$ & $\begin{array}{l}\text { Ensayo } \\
\text { clínico }\end{array}$ & $\begin{array}{l}\text { Pt: } \\
\mathrm{N}=12 \\
\text { Gsic: } 12\end{array}$ & Francia & $\begin{array}{l}\text { NPD } \\
\text { No consta }\end{array}$ & $\begin{array}{l}\text { No consta } \\
0,05 \mathrm{mg} / \mathrm{kg} / \mathrm{dia} \\
\text { Hormona del } \\
\text { crecimiento }\end{array}$ & $\begin{array}{l}49 \text { días: } \\
\text { ( } 3 \\
\text { semanas } \\
1 \text { semana } \\
\text { descanso } \\
3 \\
\text { semanas) }\end{array}$ & $\begin{array}{l}\text { En pacientes con NPD } \\
\text { y SIC, con una dieta } \\
\text { occidental hiperfágica, } \\
\text { se observó una mejora } \\
\text { significativa de la absorción } \\
\text { intestinal. }\end{array}$ \\
\hline $\begin{array}{l}\text { Scolapio } \\
\text { et al. } \\
2002(16)\end{array}$ & $\begin{array}{l}\text { Ensayo } \\
\text { clínico }\end{array}$ & $\begin{array}{l}\text { Pt: } \\
\mathrm{N}=5 ; 3 \mathrm{M} / 2 \mathrm{H} \\
\text { Edad }=61 \\
\text { años } \\
\text { Gsic:2 }\end{array}$ & EEUU & $\begin{array}{l}\text { NPD/cíclica } \\
\text { No consta }\end{array}$ & $\begin{array}{l}\text { Diaria, } 10 \\
\text { horas, } 1,5 \text { litros } \\
(25 \mathrm{kcal} / \mathrm{kg}) \text { día } \\
\text { No consta }\end{array}$ & $\begin{array}{l}\text { Mediana } \\
\text { de } 23 \\
\text { meses } \\
\text { (de } 4 \text { a } 60 \\
\text { meses) }\end{array}$ & $\begin{array}{l}\text { La calidad del sueño } \\
\text { no parecía verse afecta } \\
\text { negativamente por la } \\
\text { infusión cíclica de NPD }\end{array}$ \\
\hline $\begin{array}{l}\text { O'Keefe } \\
\text { et al. } 1994 \\
\text { (17) }\end{array}$ & Comparativo & $\begin{array}{l}\text { Pt: } \\
\mathrm{N}=41 \\
\text { Gsic: } 30 \\
\text { Gotros: } 11\end{array}$ & EEUU & $\begin{array}{l}\text { NPD } \\
\text { No consta }\end{array}$ & No consta & $\begin{array}{l}\text { Rango de } \\
\text { 1 a 15,5 } \\
\text { años } \\
\text { Promedio: } \\
78,6 \\
\text { meses }\end{array}$ & $\begin{array}{l}\text { La sepsis por catéter no } \\
\text { estaba invariablemente } \\
\text { asociada con la } \\
\text { alimentación intravenosa } \\
\text { a largo plazo, pero una } \\
\text { combinación de factores } \\
\text { del huésped (enfermedad } \\
\text { de Crohn, yeyunostomía } \\
\text { y trombosis de la vena } \\
\text { central) y factores } \\
\text { ambientales (técnica } \\
\text { de maneje del catéter) } \\
\text { aumentaban el riesgo de } \\
\text { sepsis recurrente. }\end{array}$ \\
\hline $\begin{array}{l}\text { Staun et al. } \\
1994(18)\end{array}$ & $\begin{array}{l}\text { Ensayo } \\
\text { clíico }\end{array}$ & $\begin{array}{l}\text { Pt: } \\
\mathrm{N}=15 \\
\text { Edad media: } \\
36 \text { años } \\
(23-69)\end{array}$ & Dinamarca & $\begin{array}{l}\text { NPD } \\
\text { No consta }\end{array}$ & No costa & 62 meses & $\begin{array}{l}\text { Los pacientes con } \\
\text { NPD tenian una baja } \\
\text { densidad mineral ósea } \\
\text { y que la pérdida ósea } \\
\text { continúa durante una NPD } \\
\text { prolongada. }\end{array}$ \\
\hline$=$ Pobla & 1. $\mathrm{Cr}=0$ & (Ipulat & & & & & SIC = Síndrome de \\
\hline
\end{tabular}

El acuerdo sobre la pertinencia de los estudios seleccionados, entre los dos evaluadores, calculado mediante el índice Kappa fue del 80\% ( $p=0,01)$.

Los artículos elegidos presentaron una obsolescencia, según el Índice de Burton Kebler (IBK), igual a 14,50 años, con un Índice de Price (IP) del 25,00\%. El año con mayor número de trabajos publicados fue 1994, con dos publicaciones $(17,18)$. 
Al evaluar la calidad de los artículos seleccionados para la revisión, mediante el cuestionario CONSORT, las puntuaciones oscilaron entre mínimo de 6,25 (sobre 17 ítems) y máximo de 16,50 (sobre 24 ítems) con mediana igual a 10 (tabla 2).

Tabla 2. Análisis de la calidad metodológica de los estudios a través de los 25 ítems de valoración de la guía CONSORT

\begin{tabular}{|c|c|c|c|c|c|c|c|c|}
\hline ఏ & 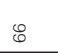 & in & $\begin{array}{l}\stackrel{0}{\mathcal{U}} \\
\tilde{U}\end{array}$ & f & 8 & $\stackrel{m}{\stackrel{m}{N}}$ & $\begin{array}{l}\infty \\
c^{\circ} \\
\rho^{-}\end{array}$ & $\begin{array}{l}\tilde{D} \\
\tilde{m}\end{array}$ \\
\hline ङूँّ & $\begin{array}{l}\stackrel{d}{N} \\
\infty \\
\stackrel{5}{\leftarrow}\end{array}$ & $\underset{\underline{N}}{\stackrel{N}{N}}$ & 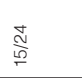 & $\sum_{\infty}^{\hat{L}}$ & $\begin{array}{l}\stackrel{J}{J} \\
\tilde{\omega} \\
\emptyset \\
-\end{array}$ & $\begin{array}{l}\stackrel{d}{N} \\
\stackrel{N}{N} \\
\stackrel{N}{n}\end{array}$ & 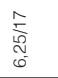 & $\underset{\stackrel{N}{N}}{\stackrel{N}{N}}$ \\
\hline$\stackrel{2}{2}$ & - & $\circ$ & $\circ$ & z & - & $\circ$ & z & $\circ$ \\
\hline ก & $\circ$ & $\circ$ & $\circ$ & z & $\circ$ & $\circ$ & $\Sigma$ & $\circ$ \\
\hline$\approx$ & - & ○ & - & 文 & $\circ$ & $\circ$ & z & $\circ$ \\
\hline ส & - & - & - & 0 & 0 & 0 & ○ & o \\
\hline$\overline{\mathrm{N}}$ & - & - & - & - & - & + & - & - \\
\hline ลิ & - & - & - & $\circ$ & $\circ$ & $\circ$ & - & $\circ$ \\
\hline$\stackrel{+}{-}$ & - & $\circ$ & - & $\circ$ & - & 0 & - & $\circ$ \\
\hline$\stackrel{\infty}{\leftarrow}$ & $\frac{\pi}{z}$ & $\frac{\pi}{z}$ & $\frac{\pi}{z}$ & $\frac{\pi}{z}$ & $\frac{\pi}{z}$ & $\frac{\pi}{z}$ & $\frac{\pi}{z}$ & $\frac{\pi}{z}$ \\
\hline$=$ & $\circ$ & - & - & $\stackrel{\llcorner}{0}$ & $\circ$ & $\circ$ & $\circ$ & $\circ$ \\
\hline$\stackrel{\circ}{\circ}$ & $\stackrel{\llcorner}{0}$ & $\circ$ & $\circ$ & - & - & + & $\stackrel{\circ}{0}$ & $\stackrel{\circ}{0}$ \\
\hline$\stackrel{2}{\circ}$ & - & - & - & - & - & - & 。 & - \\
\hline \pm & $\circ$ & ○ & - & $\circ$ & $\circ$ & $\circ$ & $\stackrel{\llcorner}{0}$ & $\stackrel{0}{\circ}$ \\
\hline 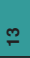 & $\stackrel{\text { ? }}{0}$ & - & - & $\circ$ & - & 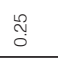 & $\stackrel{\stackrel{\sim}{N}}{\circ}$ & $\begin{array}{l}\stackrel{\llcorner}{2} \\
\stackrel{0}{0}\end{array}$ \\
\hline$\simeq$ & - & $\stackrel{0}{\circ}$ & - & $\stackrel{0}{\circ}$ & $\stackrel{\circ}{\circ}$ & $\stackrel{0}{\circ}$ & ○ & $\stackrel{0}{\circ}$ \\
\hline$=$ & $\stackrel{\llcorner}{0}$ & $\circ$ & 0 & ¿ & $\stackrel{\circ}{\circ}$ & ○ & \& & $\circ$ \\
\hline$ㅇ$ & $\stackrel{m}{0}$ & $\circ$ & $\circ$ & ¿ & - & $\circ$ & $\frac{\pi}{z}$ & $\circ$ \\
\hline 0 & $\circ$ & $\circ$ & $\circ$ & $\frac{\pi}{z}$ & - & $\circ$ & $\frac{\pi}{z}$ & $\circ$ \\
\hline$\infty$ & - & - & $\stackrel{\circ}{\circ}$ & 文 & - & $\stackrel{\llcorner}{0}$ & 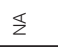 & $\circ$ \\
\hline$\wedge$ & $\circ$ & $\circ$ & $\circ$ & $\circ$ & - & $\circ$ & $\circ$ & $\circ$ \\
\hline 0 & $\stackrel{\llcorner}{0}$ & - & $\stackrel{\circ}{\circ}$ & $\stackrel{\llcorner}{0}$ & - & $\stackrel{\circ}{0}$ & $\stackrel{\llcorner}{0}$ & $\stackrel{\circ}{0}$ \\
\hline ـ & - & - & - & - & - & - & $\circ$ & - \\
\hline+ & - & $\stackrel{2}{0}$ & $\circ$ & $\stackrel{\llcorner}{0}$ & - & $\circ$ & $\circ$ & $\circ$ \\
\hline$m$ & - & $\stackrel{0}{\circ}$ & - & $\stackrel{\llcorner}{0}$ & - & $\stackrel{\llcorner}{0}$ & $\stackrel{\circ}{\circ}$ & $\stackrel{\circ}{\circ}$ \\
\hline N & - & - & + & + & - & $\stackrel{\mathscr{0}}{0}$ & + & - \\
\hline- & $\stackrel{0}{0}$ & ס & - & $\stackrel{0}{0}$ & $\stackrel{\circ}{\circ}$ & $\begin{array}{l}0 \\
0\end{array}$ & $\circ$ & 0 \\
\hline & 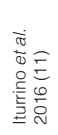 & 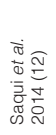 & 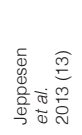 & 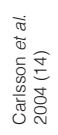 & 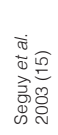 & 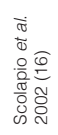 & 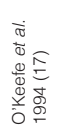 & 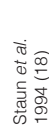 \\
\hline
\end{tabular}


Los trabajos revisados, fueron 2 estudios comparativos $(14,17)$ y 6 ensayos clínicos (11$13,15,16,18)$; desarrollados 4 de ellos en Europa $(13-15,18)$, 3 en los EE.UU. $(11,16,17)$ y 1 en Canadá (12); estando todos redactados en inglés.

La población a incluida, toda ella adulta, era de más de 50 años salvo el estudio de Staun et al. (18), que tenía media de 36 años (23 a 69) y el trabajo que más personas incluyó fue el de Jeppesen et al. (13) $(\mathrm{N}=86)$.

El tipo de nutrición empleada fue NPD estándar, salvo en el estudio de Scolapio et al. (16), en el que se empleó NPD cíclica. La frecuencia de su administración fue principalmente diaria, si bien esta solo figuraba con precisión en 3 de los 8 artículos $(11,12,16)$. Y, en otros 3 se observó que existió un fármaco asociado a la NPD: en 2 era teduglutida $(11,13)$ y en 1 fue la hormona del crecimiento (15).

El periodo de seguimiento de los estudios incluidos en esta revisión osciló entre un mínimo de 49 días (15) y un máximo con promedio de 78,6 meses (17).

En ningún trabajo se indicó el ámbito donde se desarrolló la intervención (urbano o rural), tampoco constaba si existía fluidoterapia asociada a la intervención principal (NPD) y se echó en falta en los resultados de los trabajos revisados la división de las observaciones por sexo.

\section{Resultados relacionados con la calidad de vida}

Entre las consecuencias derivadas de la administración de NPD en personas con SIC 3 estudios ofrecieron resultados relacionados con la calidad de vida $(12,13,16)$.

Saqui et al. (12), concluyeron que la de calidad de vida, medida con el cuestionario SF-36, en los pacientes que usaban bomba portátil era mejor que si la bomba estaba montada en poste.

En las investigaciones de Jeppesen et al. (13) y de Scolapio et al. (16), no existió diferencias, en relación a la calidad de vida entre los grupos analizados. El primero investigó diferencias según la utilización de un fármaco (teduglutida) versus placebo y el segundo analizó calidad de sueño según uso de NPD estándar frente a cíclica.

\section{Efectos por utilización de un fármaco asociado a la NPD}

Los fármacos utilizados en tres artículos fueron en 2 ocasiones la teduglutida $(11,13)$ y en 1 fue la hormona del crecimiento (15).

Iturrino et al. (11), indicaron el beneficio del uso de la teduglutida para el equilibrio de líquidos y la absorción de monosacáridos. Pero, constataron un retraso en el tránsito intestinal sin efecto sobre el vaciado gástrico o la permeabilidad de la mucosa. Jeppesen et al. (13), como se ha indicado en el apartado anterior no vieron asociación, en relación a la calidad de vida, por el uso, o no, de este fármaco.

Seguy et al. (15), mediante la asociación de la hormona del crecimiento a la NPD, comprobaron que existió una mejora significativa de la absorción intestinal.

\section{Complicaciones por la administración de la NPD}

En la revisión se incluyeron 3 artículos que presentaron en sus resultados algún tipo de complicación derivada del uso de la NPD $(14,17,18)$.

O'Keefe et al. (17), afirmaron que, en la NPD a largo plazo, una combinación de factores del huésped (enfermedad de Crohn, yeyunostomía y trombosis de la vena central) y factores ambientales (técnica de manejo del catéter) aumentaban el riesgo de sepsis recurrente. Staun et al.(18), 
aseveraron que los pacientes con NPD tenían una baja densidad mineral ósea y que la pérdida ósea continuaba durante una NPD prolongada.

Por el contrario, Carsson et al. (14), no vieron diferencias e indicaron que la mayoría de los pacientes con NPD mantuvieron una condición clínica estable dentro de los límites normales de peso corporal e índice de masa corporal y tampoco se alteró ni la masa libre de grasa ni el agua corporal total.

\section{DISCUSIÓN}

Al realizar la revisión de los diferentes artículos se ha podido comprobar que el estudio de las consecuencias de la administración de NPD en adultos con SIC es un tema poco tratado y que, por tanto, actualmente no se conocen resultados concluyentes.

A pesar de la importancia del tema que se revisó, se encontraron pocos artículos relacionados que presentasen las características propicias para ser analizados, dado que un gran número de ellos fueron descartados por no tratarse de estudios de alta evidencia (no eran ensayos clínicos o estudios comparativos) o por no cumplir con las características de edad de los pacientes (adultos). En este sentido, dejar constancia de la ausencia de revisiones sistemáticas anteriores sobre el objetivo en cuestión, a excepción de un trabajo de Buchholz et al., de 2015, sobre concepción, embarazo y lactancia en el SIC que requiere NPD.

La obsolescencia de los artículos seleccionados fue considerablemente mayor con respecto a otros trabajos del área de las ciencias de la nutrición, donde los datos tanto del IBK como del IP son mucho más actuales $(19,20)$.

Como cabría esperar las puntuaciones alcanzadas en la tabla CONSORT presentaron una tendencia favorable conforme más recientes fueron las publicaciones (21). Esto es debido a la poca exigencia que se requería para la difusión de artículos científicos antes del año 2010, año en el que se publicó esta declaración (22). La evaluación de la calidad de los estudios incluidos en este trabajo no resultó muy elevada a pesar de tratarse de diseños robustos, de todos modos no se eliminó ningún artículo dado el bajo número de ellos.

El ceñir la revisión a ensayos clínicos y estudios comparativos se debió a la búsqueda de una consistente relación causa-efecto, ya que el vínculo existente entre la NPD y el SIC se está volviendo una cuestión importante y, como se comprobó, poco estudiada (23).

Era predecible la filiación norte-americana o europea y que se encontrasen trabajos principalmente en lengua inglesa, este idioma es el elegido para la publicación de la mayoría de los artículos ya que hacerlo en otra lengua distinta resulta negativo para la visibilidad. Asimismo, el número de revistas anglófonas contenidas en las principales bases de datos bibliográficas es muy elevado y publicar en ellas facilita la citación (24).

El bajo número de personas adultas reclutadas en las investigaciones era predecible. Este fracaso intestinal está catalogado como enfermedad rara por su baja prevalencia lo que dificulta el poder disponer de cohortes suficientes para realizar ensayos clínicos robustos (25).

Los resultados que relacionaban la calidad de vida con el uso de una bomba portátil se deben principalmente a que las personas que cumplen criterios para recibir NPD también agradecen la independencia. La bomba portátil permite a estas personas, que ya sufrieron un cambio importante en su estilo de vida, lograr la libertad deseada y motiva la aceptación de la NPD (26). Esta conformidad es realmente importante, pues el tratamiento puede durar toda la vida e impone severas restricciones en el quehacer diario (27). Afortunadamente, desde hace ya tiempo, hay muchas bombas portátiles compactas disponibles que hacen que la terapia en el hogar sea más aceptable 
y las empresas de asistencia a domicilio suelen ser flexibles a la hora de trabajar con las familias cuando viajan (28).

Es cierto que la NPD tiene un impacto significativo en la calidad de vida, aún los resultados son generalmente buenos y la supervivencia está determinada principalmente por la enfermedad subyacente (29).

El beneficio del uso de la teduglutida para el equilibrio de líquidos y la absorción de monosacáridos ya había sido comunicado por el mismo autor en un trabajo anterior donde afirmaba que este principio era intestinotrófico y sugería efectos pro-absorbentes en pacientes con SIC (30). En el mismo sentido, Vipperla y O'Keefe (31), señalaron que este fármaco, si bien debía ser mejor evaluado para la práctica clínica, ofrecía un nuevo enfoque específico para el manejo de la insuficiencia intestinal asociada al SIC. Kochar y Herfarth (32), en una revisión publicada en 2018, ultimaron que por los datos disponibles, aunque limitados debido al pequeño número de pacientes en los estudios realizados hasta ahora, que la teduglutida parece ser segura para el uso en pacientes con insuficiencia intestinal que dependen del soporte parenteral.

La mejora significativa de la absorción intestinal por la unión de la hormona del crecimiento a la NPD vista en los resultados de esta revisión son, según Guo et al. (33), son controvertidos. Aun así, estos mismos autores observaron que tras semanas de terapia de rehabilitación intestinal con esta hormona mejoraba significativamente la absorción intestinal en el SIC, aunque en este hallazgo tiene un importante papel la glutamina (15).

Las complicaciones mencionadas por O'Keefe et al. (17), por mezcla de factores del huésped y factores ambientales podrían disminuirse mediante la adopción de medidas preventivas tempranas (mantenimiento de la hidratación, anticoagulación a largo plazo, descontaminación del centro del catéter y buena técnica de manejo del catéter). A pesar de ello, para la mayoría de los pacientes, la opción preferida es la terapia intravenosa domiciliaria que incluye nutrición parenteral, con una buena probabilidad de supervivencia a largo plazo (34).

La baja densidad ósea que comentaron Staun et al. (18), no fue observada por Cohen-Solal et al. (35), que vieron que la nutrición parenteral no tuvo ningún efecto perjudicial sobre el hueso cortical y en realidad mejoró el hueso trabecular en pacientes cuya enfermedad intestinal comenzó después de los 21 años. Recordar que como ya se ha comentado en esta revisión los resultados existentes hasta el momento hay que tratarlos con precaución debido al pequeño número de pacientes estudiados hasta el momento y que estamos ante una enfermedad de muy baja prevalencia.

Posibles limitaciones al estudio: La principal limitación sería el escaso número de estudios recuperados que impide tener un claro conocimiento entre la intervención (NPD) y sus consecuencias en el SIC, pero hoy por hoy es complicado, como ya se ha explicado, disponer de un mayor número de estudios de alta evidencia.

La diferencia entre el número de artículos conseguidos entre la búsqueda bibliográfica y los realmente válidos se debió a la base de datos Scopus, la cual no dispone de Thesaurus, por lo que no permite el uso de Descriptores para la búsqueda precisa de los ensayos, aumentando la aparición de "ruido" en los documentos recogidos tras la pesquisa (21). Por otro lado, a pesar de recuperarse varios trabajos con el límite "Adulto", una vez conseguido el artículo completo, se observaba la aparición de menores de edad en dicho estudio, siendo uno de los criterios de exclusión. Como, por ejemplo, en las publicaciones de Ławiński et al. (36), y de Galandiuk et al., (37).

Es probable que el cuestionario CONSORT no fuera el más indicado para evaluar la calidad de los artículos revisados, sin embargo, se ha tratado de paliar dicha limitación, pues no hay constancia de que exista ningún otro cuestionario que evalúe tanto los estudios comparativos como los ensayos clínicos. 
La no comunicación del ámbito de desarrollo de la intervención, la no indicación relacionada con la fluidoterapia y la carencia de segregación por sexo deben considerarse también importantes limitaciones, ya que no permitieron conocer esta valiosa información.

Por todo lo anteriormente expresado, se podría concluir:

Los trabajos revisados presentaban una obsolescencia superior a lo esperado y estaban redactados preferentemente en inglés.

Se probó mejor calidad de vida en los pacientes que usaban bomba portátil, el uso de teduglutida resultó beneficioso para el equilibrio de líquidos y la absorción de monosacáridos, pero produjo un retraso en el tránsito intestinal sin efecto sobre el vaciado gástrico o la permeabilidad de la mucosa. La asociación de la hormona del crecimiento con la NPD mejoró la absorción intestinal.

En los enfermos con SIC y NPD a largo plazo, una combinación de factores del huésped (enfermedad de Crohn, yeyunostomía y trombosis de la vena central) y factores ambientales (técnica de manejo del catéter) aumentaban el riesgo de sepsis recurrente y además tenían una baja densidad mineral ósea que continuaba en la NPD prolongada.

Serían necesarias futuras investigaciones, con una adecuada población, que aclaren la relación directa de los efectos de la NPD en adultos con SIC.

\section{BIBLIOGRAFÍA}

1. Kappus M, Diamond S, Hurt RT, Martindale R. Intestinal failure: new definition and clinical implications. Curr Gastroenterol Rep. 2016;18(9):48. DOI: 10.1007/s11894-016-0525-x; PMID: 27447791

2. Grau Carmona T, Bonet Saris A, Fernández Ortega F. Nutrición artificial en la insuficiencia intestinal: síndrome de intestino corto - Enfermedad inflamatoria intestinal. Nutr Hosp. 2005;20(Supl 2):S31-3. PMID: 15981847

3. Gonzalez Aguilera B, Olveira G, García Luna PP, Pereira Cunill JL, Luengo LM, Pérez de la Cruz A, et al. Documento de consenso de expertos de Andalucía y Extremadura sobre la nutrición parenteral domiciliaria. Nutr Hosp. 2017;34(4):784-91. DOI: 10.20960/nh.881; PMID: 29094999

4. Shatnawei A, Parekh NR, Rhoda KM, Speerhas R, Stafford J, Dasari V, et al. Intestinal failure management at the Cleveland Clinic. Arch Surg Chic III 1960. 2010;145(6):521-7. DOI: 10.1001/ archsurg.2010.103; PMID: 20566970

5. Winkler MF, Smith CE. Clinical, social, and economic impacts of home parenteral nutrition dependence in short bowel syndrome. JPEN J Parenter Enteral Nutr. 2014;38(Suppl 1):S32-7. DOI: 10.1177/0148607113517717; PMID: 24418898

6. Naber AHJ, Rings EHHM, George E, Tolboom JJM, Jonkers C, Sauerwein HP. Treatment of intestinal failure by total parenteral nutrition at home in children and adults. Ned Tijdschr Geneeskd. 2005;149(8):385-90. PMID: 15751316

7. Bhatia J, Gates A, Parish A. Medical management of short gut syndrome. J Perinatol Off J Calif Perinat Assoc. 2010;30 Suppl:S2-5. DOI: 10.1038/jp.2010.100; PMID: 20877403

8. Reinshagen K, Adams R, Trunk M, Wessel LM. The chronic liver disease in patients with short bowel syndrome: etiology and treatment. Minerva Pediatr. 2009;61(3):273-81. PMID: 19461571

9. Wanden-Berghe C, Sanz-Valero J. Systematic reviews in nutrition: standardized methodology. Br J Nutr. 2012;107(Suppl 2):S3-7. DOI: 10.1017/S0007114512001432; PMID: 22591902 
10. Begg C, Cho M, Eastwood S, Horton R, Moher D, Olkin I, et al. Improving the quality of reporting of randomized controlled trials: The CONSORT statement. JAMA. 1996;276(8):637-9. DOI: 10.1001/jama.1996.03540080059030; PMID: 8773637

11. Iturrino J, Camilleri M, Acosta A, O'Neill J, Burton D, Edakkanambeth Varayil J, et al. Acute effects of a glucagon-like peptide 2 analogue, teduglutide, on gastrointestinal motor function and permeability in adult patients with short bowel syndrome on home parenteral nutrition. JPEN J Parenter Enteral Nutr. 2016;40(8):1089-95. DOI: 10.1177/0148607115597644; PMID: 26223941

12. Saqui O, Fernandes G, Allard JP. Quality of life analysis during transition from stationary to portable infusion pump in home parenteral nutrition patients: a Canadian experience. Nutr Clin Pract. 2014;29(1):131-41. DOI: 10.1177/0884533613516129; PMID: PMID: 24347531

13. Jeppesen PB, Pertkiewicz M, Forbes A, Pironi L, Gabe SM, Joly F, et al. Quality of life in patients with short bowel syndrome treated with the new glucagon-like peptide-2 analogue teduglutide--analyses from a randomised, placebo-controlled study. Clin Nutr. 2013;32(5):713-21. DOI: 10.1016/j.clnu.2013.03.016; PMID: 23587733

14. Carlsson E, Bosaeus I, Nordgren S. Body composition in patients with short bowel syndrome: an assessment by bioelectric impedance spectroscopy (BIS) and dual-energy absorptiometry (DXA). Eur J Clin Nutr. 2004;58(6):853-9. DOI: 10.1038/sj.ejcn.1601886; PMID: PMID: 15164105

15. Seguy D, Vahedi K, Kapel N, Souberbielle J-C, Messing B. Low-dose growth hormone in adult home parenteral nutrition-dependent short bowel syndrome patients: a positive study. Gastroenterology. 2003;124(2):293-302. DOI: 10.1053/gast.2003.50057; PMID: 12557135

16. Scolapio JS, Savoy AD, Kaplan J, Burger CD, Lin S-C. Sleep patterns of cyclic parenteral nutrition, a pilot study: are there sleepless nights? JPEN J Parenter Enteral Nutr. 2002;26(3):214-7. DOI: 10.1177/0148607102026003214; PMID: 12005465

17. O'Keefe SJ, Burnes JU, Thompson RL. Recurrent sepsis in home parenteral nutrition patients: an analysis of risk factors. JPEN J Parenter Enteral Nutr. 1994;18(3):256-63. DOI: 10.1177/0148607194018003256; PMID: 8065002

18. Staun M, Tjellesen L, Thale M, Rannem T, Schaadt O, Jarnum S. Bone mineral content in patients on home parenteral nutrition. Clin Nutr. 1994;13(6):351-5. DOI: 10.1016/0261-5614(94)90024-8; PMID: 16843413

19. Quesada-Risueño P, Sanz Valero J, Wanden-Berghe C. Análisis bibliométrico de la producción científica existente en la base de datos bibliográfica MEDLINE sobre la fibra dietética. Rev Esp Nutr Humana Dietética. 2017;21(1):29-38. DOI: 10.14306/renhyd.21.1.275

20. Sanz-Valero J, Wanden-Berghe C. Análisis bibliométrico de la producción científica, indizada en MEDLINE, sobre los servicios de salud proporcionados por las unidades de hospitalización a domicilio. Hosp Domic. 2017;1(1):21. DOI: 10.22585/hospdomic.v1i1.3

21. Domingo Pena V, Sanz Valero J. Efectos de la inmunoterapia en el paciente adulto en el domicilio: revisión sistemática. Hosp Domic. 2018;2(2):15-25. DOI: 10.22585/hospdomic.v2i2.40

22. Eldridge SM, Chan CL, Campbell MJ, Bond CM, Hopewell S, Thabane L, et al. CONSORT 2010 statement: extension to randomised pilot and feasibility trials. BMJ. 2016;355:15239. DOI: 10.1136/bmj.i5239; PMID: 27777223

23. Aracil-Lavado E, Wanden-Berghe C, Sanz-Valero J. Evaluación de la calidad de vida según el estado nutricional del paciente paliativo adulto: revisión sistemática. Hosp Domic. 2017;1(4):199210. DOI: 10.22585/hospdomic.v1i4.27 
24. Franco-López AA, Sanz-Valero J, Culebras JM. Publicar en castellano, o en cualquier otro idioma queno sea inglés, negativo para el factor de impacto. J Negat No Posit Results. 2016;2(2):65-70. DOI: 10.19230/jonnpr.2016.1.2.1005

25. Instituto de Salud Carlos III (ISCIII). Portal de registro de enfermedades raras [página Web]. [citado 20 de octubre de 2018]. Disponible en: https://bit.ly/2Ak16jC

26. Boutin J, Hagan E. Patients' preference regarding portable pumps. J Intraven Nurs. 1992;15(4):230-2. PMID: 1500992

27. Baxter JP, Fayers PM, McKinlay AW. A review of the instruments used to assess the quality of life of adult patients with chronic intestinal failure receiving parenteral nutrition at home. $\mathrm{Br} \mathrm{J}$ Nutr. 2005;94(5):633-8. DOI: 10.1079/BJN20051533; PMID: 16277762

28. Bilodeau JA. A home parenteral nutrition program for infants. JOGNN. 1995;24(1):72-6. PMID: 7714642

29. Bielawska B, Allard JP. Parenteral Nutrition and Intestinal Failure. Nutrients. 2017;9(5). DOI: 10.3390/nu9050466; PMID: 28481229

30. Jeppesen PB, Gilroy R, Pertkiewicz M, Allard JP, Messing B, O'Keefe SJ. Randomised placebocontrolled trial of teduglutide in reducing parenteral nutrition and/or intravenous fluid requirements in patients with short bowel syndrome. Gut. julio de 2011;60(7):902-14. DOI: 10.1136/ gut.2010.218271; PMID: 21317170

31. Vipperla K, O'Keefe SJ. Study of teduglutide effectiveness in parenteral nutrition-dependent short-bowel syndrome subjects. Expert Rev Gastroenterol Hepatol. noviembre de 2013;7(8):6837. DOI: 10.1586/17474124.2013.842894; PMID: 24134154

32. Kochar B, Herfarth $\mathrm{HH}$. Teduglutide for the treatment of short bowel syndrome - a safety evaluation. Expert Opin Drug Saf. julio de 2018;17(7):733-9. DOI: 10.1080/14740338.2018.1483332; PMID: 29848084

33. Guo M, Li Y, Li J. Effect of growth hormone, glutamine, and enteral nutrition on intestinal adaptation in patients with short bowel syndrome. Turk J Gastroenterol. 2013;24(6):463-8. DOI: 10.4318/tjg.2013.0555; PMID: 24623283

34. Harrison E, Allan P, Ramu A, Vaidya A, Travis S, Lal S. Management of intestinal failure in inflammatory bowel disease: small intestinal transplantation or home parenteral nutrition? World J Gastroenterol. 2014;20(12):3153-63. DOI: 10.3748/wjg.v20.i12.3153; PMID: 24696601

35. Cohen-Solal M, Baudoin C, Joly F, Vahedi K, D'Aoust L, De Vernejoul MC, et al. Osteoporosis in patients on long-term home parenteral nutrition: a longitudinal study. J Bone Miner Res. 2003;18(11):1989-94. DOI: 10.1359/jbmr.2003.18.11.1989; PMID: 14606511

36. Ławiński M, Majewska K, Fołtyn I, Gradowska A. The efficacy of alcohol-antibiotic lock therapy for treatment of catheter related bloodstream infections in patients receiving home parenteral nutrition. Pol Przegl Chir. 2015;86(12):563-8. DOI: 10.1515/pjs-2015-0002; PMID: 25803055

37. Galandiuk S, O'Neill M, McDonald P, Fazio VW, Steiger E. A century of home parenteral nutrition for Crohn's disease. Am J Surg. 1990;159(6):540-5. PMID: 1972002 